\title{
Assessment of student understanding on light interference
}

\author{
Rui Dai, ${ }^{1,2}$ Joseph C. Fritchman, ${ }^{2}$ Qiaoyi Liu, ${ }^{2}$ Yang Xiao, ${ }^{3}$ Haibo Yu, ${ }^{1, \dagger}$ and Lei Bao $\odot^{2, *}$ \\ ${ }^{1}$ School of Physics, Northeast Normal University, Changchun, Jilin 130024, China \\ ${ }^{2}$ Department of Physics, The Ohio State University, Columbus, Ohio 43210, USA \\ ${ }^{3}$ School of Physics and Telecommunication Engineering, \\ South China Normal University, Guangzhou 510006, China
}

(Received 27 May 2019; published 2 October 2019)

\begin{abstract}
Light interference is an essential topic for understanding the wavelike nature of light, however, there are limited studies on modeling and assessing students' misconceptions and learning difficulties in this area. Based on the knowledge integration modeling approach, a conceptual framework for light interference is developed and used to model student understanding and guide the development of an assessment tool on light interference. The conceptual framework provides a representation of students' reasoning pathways to clearly show their connections through different conceptual components and contextual features of problem-solving settings. This type of representation focuses on showing students' knowledge structures regarding the features of integration and fragmentation. Experts' reasoning pathways always flow through a central idea of a concept with well-established connections to a wide range of contextual features and conditions. These connections form an integrated knowledge structure, which demonstrates deep understanding. In contrast, novices often focus on surface details without linking the central idea, forming fragmented local connections that link directly between contextual features and task outcomes. As a result, novice students' problem solving often relies on memorization of formula and solutions without any deep understanding. Through testing and interviews at a large Chinese university, a light interference test (LIT) has been developed and validated. Assessment results also demonstrate that students with a strong conceptual understanding of the central idea are able to apply expertlike reasoning to familiar and novel questions regardless of the contextual details. Meanwhile, students with weaker or nonexistent understanding of the central idea often struggle when novel situations are presented. LIT provides a useful tool to measure students' conceptual understanding on light interference and probe thought pathways of students' reasoning that can further indicate students' knowledge structure and levels of deep understanding.
\end{abstract}

DOI: 10.1103/PhysRevPhysEducRes.15.020134

\section{INTRODUCTION}

A fundamental goal of science education is for students to develop a deep understanding of disciplinary core ideas such that they are able to apply the knowledge to solve complex problems in novel situations $[1,2]$. However, the lack of this deep understanding is prevalent among many students, even though they may be well versed in "traditional textbook problems" [3-7]. In part, this phenomenon is caused by traditional education methods, which often promote memorization of rules and algorithms fitting to the

\footnotetext{
Corresponding author.

bao.15@osu.edu

Corresponding author.

yuhb551@nenu.edu.cn

Published by the American Physical Society under the terms of the Creative Commons Attribution 4.0 International license. Further distribution of this work must maintain attribution to the author(s) and the published article's title, journal citation, and DOI.
}

traditional textbook problems and yield mostly lower end knowledge and skills $[8,9]$. Limited to remembering context-specific solutions with little generalization, students in turn tend to use pattern matching when solving problems and demonstrate little conceptual understanding [10].

Many studies analyzing student problem-solving behavior find that student knowledge organization is one of the key factors distinguishing experts from novices [11-18]. Novices' knowledge structures are fragmented and poorly clustered around previously encountered contexts with few links between them $[11,12,15-18]$. When solving problems, this lack of organization leads to novices relying on surface features and directly matching these features with equations and outcomes [11-13]. As a result, novices' applications of a concept are constrained to contexts that are similar to those taught in class or presented in textbooks. Therefore, they are often unable to transfer their understanding to apply the same principle to novel situations. In contrast, experts' knowledge structures are integrated and hierarchically arranged around a few core principles with well-established links connecting a wide 
range of conceptual components and contextual aspects related to the content domain $[11,12,15-18]$. These connections form a comprehensive network that links concrete contextual features with core conceptual ideas reaching deep in the abstract domain such that experts' knowledge cannot be reduced to simple sets of isolated facts or propositions. This well-integrated knowledge structure allows experts to solve problems by connecting surface features and appropriate principles to determine the optimal strategy to apply this principle in the given contexts $[11,13,14]$. Therefore, experts are able to transfer between learned domains and novel situations and can then solve problems designed with unfamiliar contexts.

The process of forming generalized and cohesive knowledge structures by incorporating novel ideas is commonly studied in "knowledge integration" [19-26]. This refers to the process of organizing knowledge into broader categories while distinguishing between similar ideas and ordering the web of connections among the ideas [19-24]. Experts' knowledge structures then become hierarchically organized around a set of central ideas, which may be prompted through a range of phenomena [19-24].

The transition of a student from a novice to an expert requires the novice to gain organization within their knowledge structure through the process of knowledge integration. A number of instruction methods have been used and proven to help students overcome fragmentation and improve students' conceptual understanding. Among these are methods such as Peer Instruction, clickers, studio learning, group discussions, learning by inquiry, etc., [27-30]. The common theme within these methods is to improve student learning by carefully targeting perceived deficits and encourage students to actively explore and discuss. Through these methods the surface, deep, and implicit connections among concepts are highlighted in such a way that encourages critical evaluation by students in varying contexts [31].

Because of the complexity of students' learning behaviors, designing an assessment that can probe deep into their knowledge structures can be challenging [21,32]. This study will experiment with an assessment approach to probe features of students' knowledge structures by designing the assessment around the central idea of a concept and probing the links between the central idea and contexts. To clearly represent the contextual and conceptual elements of a knowledge structure as well as the possible reasoning pathways connecting these elements, a modeling method of conceptual framework on light interference is developed based on the approach outlined in a recent study [33]. The conceptual framework is then used to guide the development of the assessment to specifically target the reasoning pathways that lead to various misconceptions and problemsolving difficulties. In addition, the assessment makes use of both typical (familiar) and atypical (novel) questions to measure the extent of students' ability in transferring knowledge across familiar and novel contexts, which provides useful information for inferences on students' knowledge structures. The hypothesis is that when students have well connected and integrated knowledge structures, they will be able to successfully apply their understanding to solve problems designed with both typical (familiar) and atypical (novel) contexts. Meanwhile, students with fragmented knowledge structures will perform better on questions designed with typical contexts than on questions with atypical contexts. It is important to note that the designations of typical and atypical are dependent on the instruction students received.

In this study, a conceptual framework on light interference is developed and used to guide the design of an instrument for assessment of students' understanding of the concept. Two areas of research will be conducted in this study:

- Identify student difficulties in learning light interference and analyze these difficulties with the conceptual framework and the knowledge integration perspective.

- Experiment with an assessment approach of using typical and atypical contexts to probe deep understanding and make inferences on students' knowledge structures.

\section{METHOD AND DESIGN}

\section{A. Knowledge integration and conceptual framework}

The development of the conceptual framework is based on theories of conceptual change and knowledge integration [33]. Cognitive structures of concepts are fundamentally restructured when students learn science concepts, such that networks of ideas become increasingly integrated [34,35]. A key factor in evaluating students' knowledge integration is the ability to consistently use a central idea across a range of phenomena or contexts $[23,26]$. This central idea provides an anchor point to link additional ideas and acts as the central node of a well-connected knowledge network. Furthermore, it has been demonstrated that instruction emphasizing the central ideas is productive in promoting knowledge integration, leading students to develop deeper conceptual understanding [26,33]. The conceptual framework itself is then rooted in specific aspects of these theories. First, deeply rooted nonscientific conceptions are commonly held by students [34]. Second, students' conceptions are often context dependent and exist as disconnected knowledge fragments within these contexts [36-38]. Finally, productive assessment and instruction should focus on measuring and improving the coherence of students' knowledge structures rather than expanding collections of isolated knowledge pieces.

The conceptual framework representation was developed to clearly illustrate differences in the knowledge structure regarding different states of a learner's conceptions, from a novice to an expert. Here, a learner's ideas are activated by 
and depend on contextual features. An expert links the ideas and contexts to form conceptual pathways around the central idea, with which the network of such pathway connections forms a fully integrated knowledge structure. In contrast, a novice often bypasses the central idea and develop direct links using memorized algorithms or equations among surface features of the problem and aspects of the problem solutions or outcomes [12,33,36,37]. The novice's approach may produce correct results in limited situations; however, increased complexity in the contexts and variables will quickly lead to mastery by memorization becoming unfeasible. On the other hand, relating wideranging situations to a central idea will aid in students' forming well-integrated knowledge structures to support the construction of deeper, expertlike conceptual understanding.

Creating a conceptual framework for a concept relies on first identifying the central idea associated with the concept, followed by common contexts and processes associated with the concept. Pathways can then be established among the central idea, conceptual claims, contexts, and processes, while a novice's pathways often neglect the central idea and directly link contexts with certain process outcomes and conceptual claims.

Based on the conceptual framework, assessment instruments can be designed to more accurately target students' knowledge structures. Observations of students' problemsolving behaviors (through testing, work shown, and interviews) can reveal their thinking and allow mapping links and pathways contained in their knowledge structures. The conceptual framework can then help represent, explain, and predict students' problem-solving behaviors. Additionally, it provides a guide for future interventions aimed at helping students develop the missing links that are essential for achieving a deep conceptual understanding.

\section{B. Conceptual framework of light interference}

The phenomenon of light interference is one of the core components of physical optics as it provides the first experimental evidence of light's wavelike nature. A number of studies have been conducted on students regarding students' difficulties on light interference [39-48]. Some studies developed tutorials or wave visualization methods to address these difficulties and have proven to be effective [42,46,49-51]. However, the thinking and reasoning processes underlying these misconceptions and how they arise during a student's knowledge development are not yet well understood. Using the conceptual framework approach, this study aims to demonstrate how novices' fragmented knowledge structures are associated to reasoning pathways that underlie the observed misconceptions.

In building a conceptual framework, the first step is to identify the central idea [33]. For light interference, the two key components examined in this paper are the principle of superposition and the phase difference, and how they lead to determination of interference maxima and minima, which form the central idea.

The principle of superposition describes interference between multiple waves overlapping in the same space and same time. The resulting wave at each point and time is the sum of the individual waves at that point and time. For light, this leads to measurable physical consequences in the form of bright and dark fringes, which are observed spatial distributions of average light intensity, commonly referred to as interference patterns. The examples used in this study are based on the classic double-slit interference setup and its variations, in which two monochromatic light waves of the same wavelength and a stable phase difference will interfere and create an interference pattern on a screen.

The phase difference $\Delta \phi$ measures how in sync two interfering waves are at any given point in space and time. When two waves are perfectly in phase, they constructively interfere and produce a bright fringe (a maxima of average intensity); however, when two waves are perfectly out of phase, they destructively interfere and produce a dark fringe (a minima of average intensity). Constructive and destructive interference correspond directly to phase differences of even multiples of $\pi$ and odd multiples of $\pi$, respectively. Phase differences in between these result in intensities between the maxima and minima.

In many light interference experiments, the phase difference is often a result of path length difference between the two light beams $\left(\delta=r_{2}-r_{1}\right)$, where $r$ is the geometric path length that a light takes to reach a given point. In this case, the phase difference can be calculated as $\Delta \phi=2 \pi \delta / \lambda$, in which $\lambda$ is the wavelength of the light. However, both the wavelength and path length difference are dependent on the physical situation. For example, the wavelength is dependent on the index of refraction $n$ of the media, through which the light travels. Meanwhile the path length is dependent on the physical setup of the experiment. Changes in each of these often result in changes of phase differences and altered interference patterns. Ambrose et al. [39] demonstrated that students can have difficulties in recognizing the essential role of phase difference, and simply memorize the relevant formulas with little understanding of the derivation [39]. This leads to novices being unable to correctly analyze situations with novel physical and contextual variables.

Based on the physics content and research literature on student difficulties, a conceptual framework showing the core conceptual and contextual components and different reasoning pathways is developed and illustrated in Fig. 1. The top layer component is the central idea, which builds on the core understanding of superposition of waves and its relation to phase difference. An expert usually applies this central idea, either explicitly or intuitively, to all related problems. The bottom layer components are contextual variables, which represent a wide range of context features that can be modified to create different question and task 


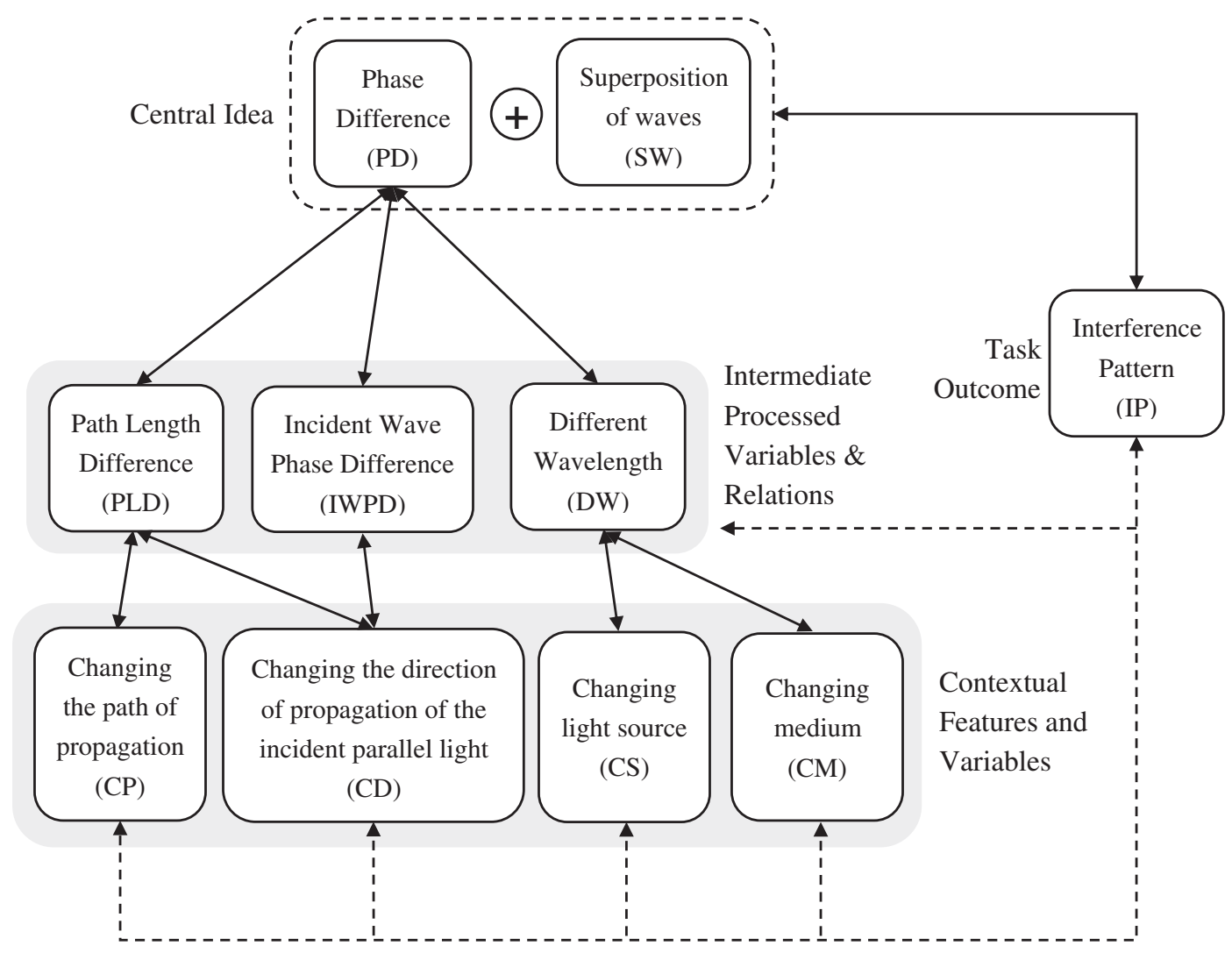

FIG. 1. Conceptual framework of light interference. Solid arrows represent the desired reasoning pathways that experts made among knowledge pieces to aid problem solving, while the dashed arrow represent the direct links between contextual features and the task outcomes that novices often made. The abbreviations within the parentheses are used to label that knowledge piece, which will be referenced in later discussion on the related reasoning.

settings. The middle layer includes locally processed intermediate outcomes as part of the context variables that are often conceptually and or mathematically logically processed results from more basic contextual features. For example, changing the path of a light beam is a contextual aspect that can be physically manipulated in an actual setting. The related outcome of such physical changes is the possible change of path difference, which needs to be identified and calculated through conceptual and mathematical processing before it can be used for further reasoning.

For most problems on the interference topic, the task is often to predict or identify the correct interference pattern, which is shown in a block on the right in Fig. 1. There are multiple arrows linking the different contextual, conceptual, and outcome components. The solid arrows represent the reasoning pathways of an expert on how to approach a problem. These all go through the central idea and link to the contexts and the outcomes, forming a well-integrated network structure (knowledge structure). On the other hand, a novice often makes short local connections between contextual layer components and the outcome. Usually, these links represent certain memorized relations and processes with little reasoning that can be extended to make connections with other components. There can also be differing levels of progressions among these novices. The weakest students may only be able to memorize the bottom layer components and make connections to the outcome, while the slightly advanced students may be able to develop local conceptual processing to transform bottom level components into intermediate level processed variables and then make connections to the task outcomes. The wide variety of novices' reasoning pathways are illustrated with dashed line arrows.

Note that all arrows are double headed in general, indicating that a connection can be initiated from either side. However, it is also possible for certain students to have only developed one direction of link activation among some of the connections, which can be used to represent certain intermediate stages of cognitive development. It is worth noting that the identified components and links for the conceptual framework shown in Fig. 1 are by no means a complete set, but rather represent the most popular ones frequently involved with the targeted concept and problem settings. Depending on the targeted level of understanding and the specific domain of content, the corresponding conceptual framework will also have varied forms and structures. 


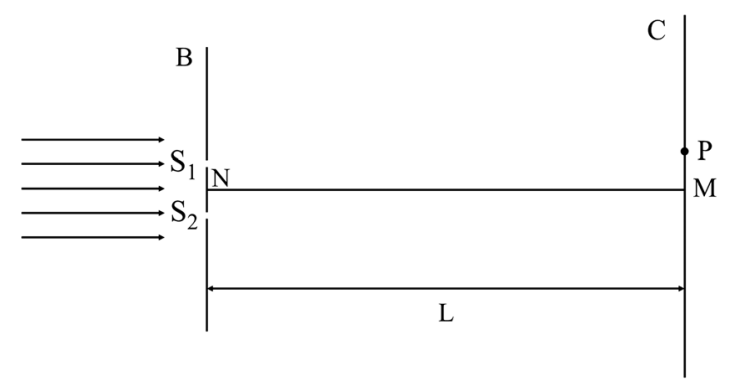

FIG. 2. Standard Young's double slit experimental setup. It is also the problem setup used in Q1-Q4, Q13, and Q14 of the assessment.

To illustrate how the conceptual framework works in practice, consider the standard Young's double slit experiment (Fig. 2): light waves are incident on a barrier with two thin slits and create an interference pattern on a screen that is placed behind the barrier. Branching from the standard setup, phase differences in Young's double slit can be altered using numerous contexts. As mentioned above, changing the path of propagation or changing the wavelength of light (e.g., switching to a different light source or placing the system in a different medium such as a different gas or glass) will alter the phase difference. Changes to the path can also occur in numerous forms such as modifying the slit positions or adjusting the angle of incident light waves.

Although the contexts above may change the exact details involved in calculating the phase difference, the central idea of light interference remains identical. The analysis of bright and dark fringes of the interference patterns still requires calculating where the waves from each slit will be in phase or out of phase with each other. Experts know this goal regardless of their familiarity with the specific context. In contrast, novices are unable to identify the core concept and often focus on contextual features and surface level details—especially when encountering novel situations.

Based on the distinction of problem-solving strategies of students with fragmented and integrated knowledge structures, one can distinguish students' understanding of light interference by asking them to solve problems designed with typical and atypical contexts. In order to help understand how the conceptual framework manifests itself within student knowledge structures, the above analysis can be summarized into three types of student behaviors which are matched with three levels of understanding. In general, there can be another level representing no understanding, which will be ignored in this discussion. In the following, the conceptual framework from Fig. 1 is used to explain the possible pathways representative for each of the three levels of understanding:

(i) Students only make direct local connections between the contextual variables (bottom layer components) and the task outcome: This is the lowest level of understanding and the knowledge structures of students in this level are mostly fragmented. As an example, students would infer the interference pattern (IP) directly from the context feature of changed path of propagation $(\mathrm{CP})$, without deriving and considering the path length difference (PLD). These students seldom reach further to the central idea, which includes the phase difference (PD) and superposition (SW). This reasoning pathway can be summarized as CP $\rightarrow$ IP (see Fig. 1 for the conceptual pathways). Students at this level typically are only able to solve certain familiar questions through memorized solutions.

(ii) Students can relate the contextual variables to the intermediate layer processed relations and variables, with which they tend to make direct connections to the task outcome without considering the central idea. This represents an intermediate level of understanding, which includes more connected links but is still fragmented without the integrated understanding that links through the central idea. As an example, students at this level can relate the fact that changing the path of propagation can lead to a change in the path length difference. However, they would infer the interference pattern (IP) directly from the path length difference, without considering the central idea. This can be summarized as a pathway of CP $\rightarrow$ PLD $\rightarrow$ IP (see Fig. 1). Students at this level are often doing well with familiar questions using a mixture of memorized solutions and locally processed intermediate relations, however, they often fail on atypical (novel) questions, which require the use of central idea.

(iii) Students can relate the contextual variables to the processed variables and relations, then to the central idea, and finally to the task outcome. This is the highest level of understanding and their knowledge structures are well connected through the central idea, just like those of the experts. With any given contextual features, students at this level can follow through the related connections all the way to the central idea, which can then be used to solve most given problems, regardless of them being typical or atypical. This reasoning pathway can be summarized as $\mathrm{CP} \rightarrow \mathrm{PLD} \rightarrow \mathrm{PD} / \mathrm{SW} \rightarrow \mathrm{IP}$ (see Fig. 1).

The established conceptual framework and the discussions of different levels of student understanding will be used to guide the development of an assessment instrument to evaluate the features of students' knowledge structures. In addition, follow-up interviews will be conducted to further examine students' thought processes and reasoning pathways.

\section{Design of the light interference test (LIT)}

Based on the conceptual framework of light interference, a light interference test is designed to probe students' deep 
conceptual understanding on light interference. These questions are designed to test students' abilities to relate contextual features to the central ideas (PD and SW) and measure the connectedness of their knowledge structures. LIT focuses on the narrow but critical field of light interference through double slits and is not directly comparable to more broad instruments such as the basic wave optics survey [42,51] or the light and optics conceptual evaluation instrument [52]. From student testing, LIT will help identify common student misconceptions and causes, which can then suggest improvements to curriculum design. The test includes 15 multiple choice questions categorized in 4 contextual sets based on the light interference conceptual framework shown in Fig. 1. The problems labeled here as typical or atypical are for the population studied in this research and may vary in other education systems depending on their instruction. However, we believe that set 1 should be completely typical for most physics students and the other sets may contain a mix. Q5 and Q6, in particular, are considered typical to the Chinese students in this study because they have explicitly covered questions where either the source or double slits shift vertically. Textbooks such as Knight's Physics for Scientists and Engineers [53] do not cover these situations, which may lead these questions to be designated as atypical when this or similar textbooks are used in a course.

Additionally, the term "zeroth bright fringe" appears in six of the questions and may hold a different definition at other institutions. Commonly, it is also referred to as the central (or brightest) fringe. This is the $m=0$ fringe such that there is zero total path length difference (and therefore zero phase difference) between the two interfering waves measured from the original source to a point on the screen. In cases where the waves leaving the double slit are not in phase (such as Q5-Q12), the total path length must be considered from the source, through each slit, and to the screen.

Set 1 contains Q1-Q4. These questions provide the phase differences or path length differences at a certain point on the screen and ask whether the image at that point is a bright or a dark fringe. This set of questions involve the most basic understanding of the key principles behind light interference and proficient students are expected to demonstrate a conceptual pathway that goes through PD $\rightarrow \mathrm{SW} \rightarrow \mathrm{IP}$.

Set 2 contains Q5-Q8. These questions alter the positions of the double slits, which in turn change the path length differences as well as the phase differences; and then ask how the zeroth bright fringe would move on the screen. Q5 and Q6 are designed with typical context scenarios frequently seen in textbook problems, while Q7 and Q8 are modified versions of Q5 and Q6 designed with atypical (novel) context scenarios rarely used in textbook problems. For these questions, proficient students are expected to demonstrate a conceptual pathway going through $\mathrm{CP} \rightarrow \mathrm{PLD} \rightarrow \mathrm{PD} \rightarrow \mathrm{SW} \rightarrow \mathrm{IP}$.
Set 3 contains Q9-Q12, which alter the direction of propagation of the incident plane wave such that it arrives at the slits at an angle. This arrangement changes the path length difference, which leads to a change of the phase difference. The questions ask how the zeroth bright fringe would move on the screen (Q9, Q10) and the phase difference at the point on the screen showing the bright fringe (Q11, Q12). All four questions in this set are considered atypical, since the context scenarios are rarely used in textbook problems. For these questions, proficient students are expected to demonstrate a conceptual pathway through $\mathrm{CD} \rightarrow \mathrm{IWPD} / \mathrm{PLD} \rightarrow \mathrm{PD} \rightarrow \mathrm{SW} \rightarrow \mathrm{IP}$.

Set 4 contains Q13-Q15 that each alter the wavelength of the incident light which leads to a change of the phase difference. The questions ask how the spacing between fringes would change. More specifically, Q13 and Q14 alter the wavelength of the light source and are considered typical, since such context scenarios are commonly used in textbook problems. For these questions, proficient students are expected to demonstrate a conceptual pathway as $\mathrm{CS} \rightarrow \mathrm{DW} \rightarrow \mathrm{PD} \rightarrow \mathrm{SW} \rightarrow \mathrm{IP}$. Q15 alters the medium through which the light travels in order to change the wavelength of the light. This question is considered atypical, since the context scenario is rarely used in textbook problems. Proficient students are expected to demonstrate a conceptual pathway through $\mathrm{CM} \rightarrow \mathrm{DW} \rightarrow$ $\mathrm{PD} \rightarrow \mathrm{SW} \rightarrow \mathrm{IP}$.

Validation and reliability evaluations of the instrument as well as exploratory factor analysis are provided in the Supplemental Material I [54], which have confirmed the separation of questions into four context sets (Q1-Q4, Q5-Q8, Q9-Q12, and Q13-Q15). LIT items are provided in the Supplemental Material II [54] along with the correct answer and a brief explanation for each item.

\section{Research procedure}

This study was conducted at a large enrollment university in China with a national ranking of top 30-40. The testing subjects are physics majors in their sophomore and junior years who have completed the introductory calculusbased mechanics and electricity and magnetism courses. During the fall semester of the second-year physics course, students first studied geometrical optics and then physical optics. The concepts of diffraction, interference, and polarization were rigorously taught in the physical optics course. Among the students who participated in this study, the sophomore students had just completed this secondyear physics course, while the juniors had taken this course in the previous year.

The test was scheduled at the beginning of the 2018 spring semester. During a $1 \mathrm{~h}$ session, students completed the LIT test in a proctored test room at their own pace. A total of 315 students took the test, with 165 sophomores and 150 juniors. 
The main purpose of this study is to investigate students' difficulties in solving double-slit interference problems with the LIT test. Statistical significances of comparisons between grade level (sophomore vs junior) and content set are determined with two-way ANOVA and further explored through student $t$ tests. Size of differences between groups of questions and groups of students are measured with Cohen's $d$ effect size.

Additionally, 41 students were randomly selected to participate in "think-out-loud interviews," where the interviewees were asked to explain their thought processes when solving these problems in as much detail as possible. Each interview lasted for about $30 \mathrm{~min}$ and was audio taped. These interviews provide an opportunity to gain more in-depth insight into how students solve problems, with a focus on distinguishing between strategies relying on memorization and those relying on deep conceptual understanding.

\section{RESULTS OF THE QUANTITATIVE STUDY}

\section{A. Students' performance varied across contexts}

Sophomore and junior students' performances across the different question sets are plotted in Fig. 3 and listed in Table I. Differences in performance between grade levels are compared using two-way ANOVA (grade level and question set). Main effects were measured for both grade level $\left[F(1,313)=36.295, p<0.001, \eta_{p}^{2}=0.104\right]$ and question set $\left[F(4,1252)=49.030, p<0.001, \eta_{p}^{2}=0.135\right]$. Directly comparing performances for each grade level reveals sophomore students with higher scores than juniors, both within each question set and overall $[t(313)=$ $6.061, p<0.001, d=0.684]$. A difference is expected because the sophomores and juniors are two distinct populations. Although they took the same course a year apart, the instructors were different, and the content emphasis and teaching methods were subject to significant variations. In addition, the one year time delay for the junior population may also be a factor impacting their performances.

However, the two populations' relative performances across the different question sets are quite similar (two nearly parallel trend lines as shown in Fig. 3). This is also

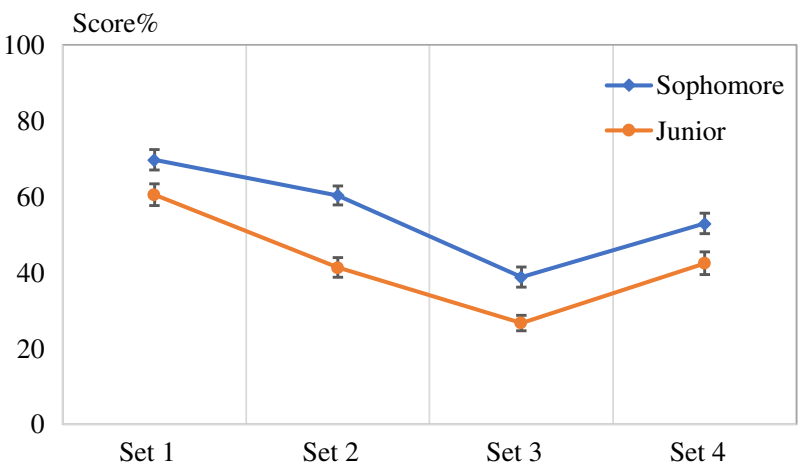

FIG. 3. Students' performance across different question sets. The error bars represent standard error.

confirmed by the ANOVA result, which shows no significant interaction between grade level and question set $\left[F(1,1252)=1.144, p=0.334, \eta_{p}^{2}=0.004\right]$. For the purposes of this study, differences between the two student groups are not the focus; instead, this study is interested in performance between question sets, regardless of class. Since there is no significant interaction between grade level and question sets, the data of sophomore and junior students are combined to study the assessment features across the different questions sets.

Effects due to question sets are first explored through one-way ANOVA which shows that students' performances are significantly different across the question sets $\left[F(4,1256)=49.087, p<0.001, \eta_{p}^{2}=0.135\right]$, except for between sets 2 and 4 [ $\left.t_{24}(314)=1.348, p=0.08\right]$, which are on the borderline. Students perform best in set 1 $\left[t_{12}(314)=5.741, p<0.001, d=0.32 ; t_{13}(314)=12.582\right.$, $\left.p<0.001, d=0.71 ; t_{14}(314)=6.828, p<0.001, d=0.38\right]$, followed by sets 2 and $4\left[t_{23}(314)=7.637, p<0.001\right.$, $\left.d=0.43 ; \quad t_{43}(314)=5.641, p<0.001, d=0.32\right], \quad$ and worst in set 3 . The differences are statistically significant among the different question sets. The question contexts involve both typical and atypical scenarios, which will be analyzed in more detail in the next section.

\section{B. Student performance on typical vs atypical questions}

In general, students' performance differences between question sets are influenced by both the content topics of

TABLE I. Summary of sophomore and junior students' performance in different question sets, and $t$-test results comparing classes. The combined total scores (combines sophomore and junior scores) are also given.

\begin{tabular}{|c|c|c|c|c|c|c|c|c|}
\hline \multirow[b]{2}{*}{ Question set } & \multicolumn{2}{|c|}{ Sophomore $(N=165)$} & \multicolumn{2}{|c|}{ Junior $(N=150)$} & \multicolumn{2}{|c|}{ Combined total } & \multirow[b]{2}{*}{$t$ test } & \multirow[b]{2}{*}{$d$} \\
\hline & Mean & (SE) & Mean & (SE) & Mean & SE & & \\
\hline 1 (Q1-Q4) & 69.70 & (2.69) & 60.50 & $(2.86)$ & 65.32 & (1.98) & $2.34 *$ & 0.26 \\
\hline 2 (Q5-Q8) & 60.30 & $(2.50)$ & 41.33 & (2.59) & 51.27 & (1.88) & $5.27 * * *$ & 0.59 \\
\hline 3 (Q9-Q12) & 38.79 & $(2.65)$ & 26.67 & (2.04) & 33.01 & (1.73) & $3.62 * * *$ & 0.40 \\
\hline 4 (Q13-Q15) & 52.93 & $(2.70)$ & 42.44 & (2.99) & 47.94 & (2.09) & $2.61 *$ & 0.29 \\
\hline Total & 55.60 & (1.50) & 42.76 & (1.49) & & & $6.06 * * *$ & 0.68 \\
\hline
\end{tabular}

Notes: $* p<0.05, * * * p<0.001$. 
TABLE II. Typical and atypical scores in each question set and statistical significance of differences.

\begin{tabular}{|c|c|c|c|c|c|c|}
\hline \multirow[b]{2}{*}{ Question set } & \multicolumn{2}{|c|}{ Typical } & \multicolumn{2}{|c|}{ Atypical } & \multirow[b]{2}{*}{$t$ test } & \multirow[b]{2}{*}{$d$} \\
\hline & Mean & (SE) $\%$ & Mean & (SE)\% & & \\
\hline 1 (Q1-Q4) & 65.32 & (1.97) & & & & \\
\hline $2(\mathrm{Q} 5-\mathrm{Q} 8)$ & 60.16 & (2.29) & 42.38 & (2.29) & $6.778 * * *$ & 0.38 \\
\hline 3 (Q9-Q12) & & & 33.01 & (1.73) & & \\
\hline 4 (Q13-Q15) & 55.40 & $(2.45)$ & 33.02 & (2.65) & $7.022 * * *$ & 0.40 \\
\hline Total & 61.55 & (1.12) & 35.69 & (1.31) & $14.456^{* * *}$ & 0.81 \\
\hline
\end{tabular}

the question sets and the contextual features, including the use of typical and atypical scenarios. Within each question set, the content topics of the questions are similar, while the contextual features may vary substantially. In question set 1 , all questions are designed with typical contexts. For question sets 2 and 4, the questions are designed with both typical (Q5-6 and Q13-14) and atypical (Q7-8 and Q15) contexts. Questions in set 3 are all with atypical contexts. Although the contextual designs are not evenly distributed across all the question sets due to constraints of content topics, the current configuration still provides an opportunity to investigate the impact of typical vs atypical contexts. The average scores of questions using typical and atypical contexts are calculated for each question set and listed in Table II.

The results show that the scores of questions with typical contexts are much higher than those with atypical contexts [mean score difference $=25.86 \%, t(314)=14.456, p<0.001$, $d=0.81]$. Within both question sets 2 and 4 , the differences between scores of questions with typical and atypical contexts are still quite substantial at the $20 \%$ level with effect sizes at the 0.40 level. Since the content topics within each question set are similar, the results of question sets 2 and 4 can be considered as primarily the outcomes of typical and atypical contexts. In addition, the variations of the mean scores within typical and atypical questions across different question sets are at the 6\%-7\% level, much smaller than the differences between typical and atypical questions (20\%$25 \%$ level). This further confirms that students' performance differences are caused more by the use of typical and atypical contexts than by content variations.

Furthermore, Fig. 4 plots students' mean scores on typical and atypical questions vs their overall scores $\left(S_{\text {total }}\right)$. The overall score (horizontal axis) can be considered as a measure of students' conceptual development level. The plots are similar to the item response curves $[53,55]$, but are used here to represent the performance differences between typical and atypical questions. From the diagram, students with overall scores lower than $20 \%$ scored with no difference between typical and atypical questions. Students at this low level of understanding were basically responding at chance, and therefore, the contexts of the questions did not make any difference. For students with overall scores between $\sim 0.25$ and $\sim 0.90$ there are significant differences between students' scores on typical and atypical questions. The performance gap between the two types of questions starts to split substantially around $S_{\text {total }}=0.40$ and then closes around $S_{\text {total }}=0.80$. This result suggests that students at intermediate level of conceptual development often have more fragmented understanding with strong context dependence so that they are able to solve questions with familiar contexts but would fail on questions with novel contexts. Meanwhile, students at higher level of conceptual understanding are able to develop more integrated knowledge structures and can transfer to solve questions with unfamiliar contexts. This result reflects a cross-sectional snap shot of a population distribution with a range of low to high level of conceptual understanding and doesn't indicate any longitudinal developmental processes. However, one can still gain valuable insights on how students' knowledge structures may evolve through actual learning development.

Summarizing these results, students perform better within contexts they are familiar with than in novel situations, as is expected with a context dependent fragmented knowledge structure. The overall concept is similarly understood as long as the context presented is familiar. Likewise, students appear to perform similarly poor when presented with an atypical situation. The results

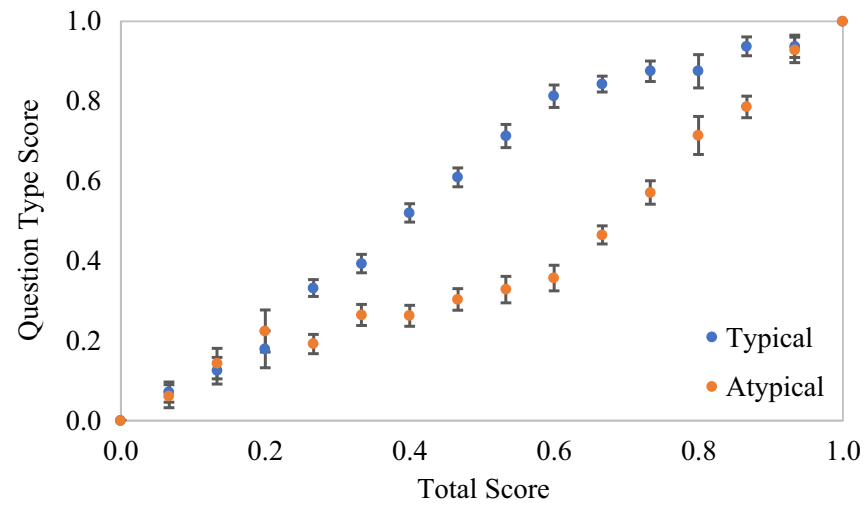

FIG. 4. Typical and atypical question scores based on total scores (with error bars denoting standard errors). The scores shown are the averages of all questions of that type (8 typical, 7 atypical, and 15 total questions). Differences are significant in the range of total scores of approximately $0.25-0.90$. 
demonstrate that how the concept is presented to students is important (comparing typical sets to other typical sets), yet the familiarity of students with the context plays a larger role in student understanding (comparing typical to atypical). The results are consistent with the predictions of student behaviors based on their conceptual developmental level and their implied knowledge structures, which are further illustrated with the outcomes shown in Fig. 4. Therefore, using typical and atypical questions appears to be a viable method to probe students' knowledge structure and level of knowledge integration. In the following section, student interviews are probed to further elicit differences in student reasoning on typical and atypical questions.

\section{RESULTS OF THE QUALITATIVE STUDY}

Overall, 41 students were interviewed to supplement the test data above and gain further understanding of student knowledge structures. These students were roughly split between the sophomore and junior classes. However, the data are combined in this section because the primary interest is the existence of misconceptions, problemsolving strategies, and knowledge structures instead of differences between the two classes.

\section{A. All typical questions (question set 1)}

Question set 1 is formed by Q1-Q4 and each question is based on a standard Young's double slit interference setup (Fig. 2). These questions ask whether bright or dark fringes appear at a point on the screen given the phase or path length difference at that point. The design of these questions aims to test students' reasoning following the conceptual pathway of PD $\rightarrow$ SW $\rightarrow$ IP. The contexts of the questions are similar to typical questions students have seen during coursework.

Based on Table I, students were generally able to answer these questions correctly. The follow-up interviews helped identify two types of student understanding: memorization of relevant formulas and deep understanding of the central ideas.
Among all the interviewed students only three demonstrated their thought processes from the pictures they drew to help analyze the superposition of two waves (examples in Fig. 5). This strategy implies that they had acquired a deep understanding of the central ideas and directly connected the phase or path difference to the superposition and interference patterns. As a robust approach, this method would allow students to correctly answer these (and other) questions without the need to memorize the formula. In fact, the formula can be easily derived based on the understanding of the central idea.

All the remaining students based their responses on remembering and applying the related equations. Their memorization focused on two textbook equations that establish conditions for constructive and destructive interference based on either the phase difference [Eq. (1)] or the path length difference [Eq. (2)]:

$$
\begin{gathered}
\Delta \varphi=\left\{\begin{array}{ll}
2 j \pi & \text { bright } \\
(2 j+1) \pi & \text { dark }
\end{array}, \quad j=0, \pm 1, \pm 2 \ldots,\right. \\
\delta=\left\{\begin{array}{ll}
j \lambda & \text { bright } \\
\left(j+\frac{1}{2}\right) \lambda & \text { dark }
\end{array}, \quad j=0, \pm 1, \pm 2 \ldots\right.
\end{gathered}
$$

Six students remembered both equations exactly and were able to "plug and chug" to correctly answer all four questions. While these students did not explicitly demonstrate knowledge of the central ideas for this set of questions, this response does not rule out the possibility that a deep understanding of the concept exists. Instead, as reflected by their responses, they found it easy to solve these problems by applying the memorized formula under these contexts regardless of the conceptual understanding.

Eleven students only remembered the path length formula but were able to correctly convert between path length difference and phase difference $(\Delta \phi=2 \pi \delta / \lambda)$. This demonstrates an approach of equation memorization combined with some relevant conceptual understanding. This group of students can be considered as having a moderate level of integration in their knowledge structure, since transferring

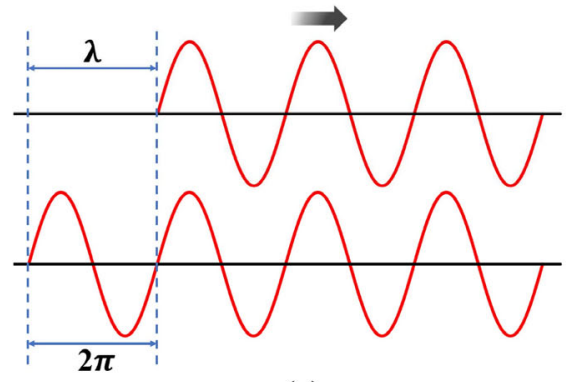

(a)

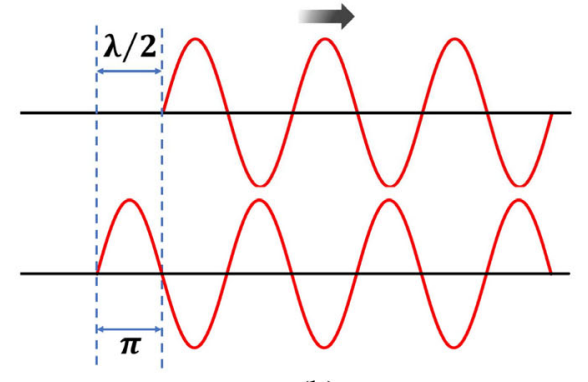

(b)

FIG. 5. (a) Constructive interference. (b) Destructive interference 
from path difference to phase difference and vice versa is an important conceptual component of the central idea.

Another 11 students could remember the basic structures of the equations such as some of the related variables, but not in the complete form. Many of these students did not recognize that half of a wavelength path difference was identical to $\pi$ phase difference, leading to inconsistent applications of what they could remember. The final 10 students remembered that the equations existed, but not their forms or what they meant. Common errors in this group included confusing phase and path length differences and which one was associated with wavelength or $\pi$. Therefore, these two groups of students represent the lower level of understanding that only contains fragmented conceptual pieces as well as the impact of forgetting.

Each of these questions was based on typical questions students see during their coursework and required minimal abstraction from memorized material or an understood concept to answer correctly. As such, it was expected that students would generally perform well. However, there still exists substantial differences in problem-solving methods between students who understand the central ideas and those who only partially understand it. Students with weaker understanding appear to more strictly follow the memorized equations and rules for each individual context. Some demonstrated additional understanding of the concept by using it to convert between path and phase difference while others demonstrated no understanding, unable to apply a remembered (or incorrectly memorized) formula.

This provides evidence showing that students, even when given familiar situations, do not possess a uniform knowledge structure. That is, each student learns a set of pathways that were activated during learning by a familiar problem, but these pathways can differ from student to student. Some of these pathways can lead directly through the central idea, which allows students to logically reason through a problem to its conclusion. However, it appears much more common for pathways to exist leading students to algorithmic application of memorized equations, which leads to difficulties when equations are incorrectly memorized or applied to inappropriate situations. Some students only form weak versions of these pathways and demonstrate significant uncertainty in their answers and reasoning.

\section{B. Mix of typical and atypical (question sets 2 and 4)}

Question sets 2 and 4 each includes both typical and atypical questions. As shown in Fig. 6, question set 2 (Q5-Q8) focuses on Young's double slit questions where the position of the slits is altered by changing distance between slits and screen (typical contexts) and by adding a second double slit positioned between the first double slit and the screen (atypical contexts). These questions were designed to test students' abilities to establish the connection $\mathrm{CP} \rightarrow \mathrm{PLD} \rightarrow \mathrm{PD} \rightarrow \mathrm{SW} \rightarrow \mathrm{IP}$.

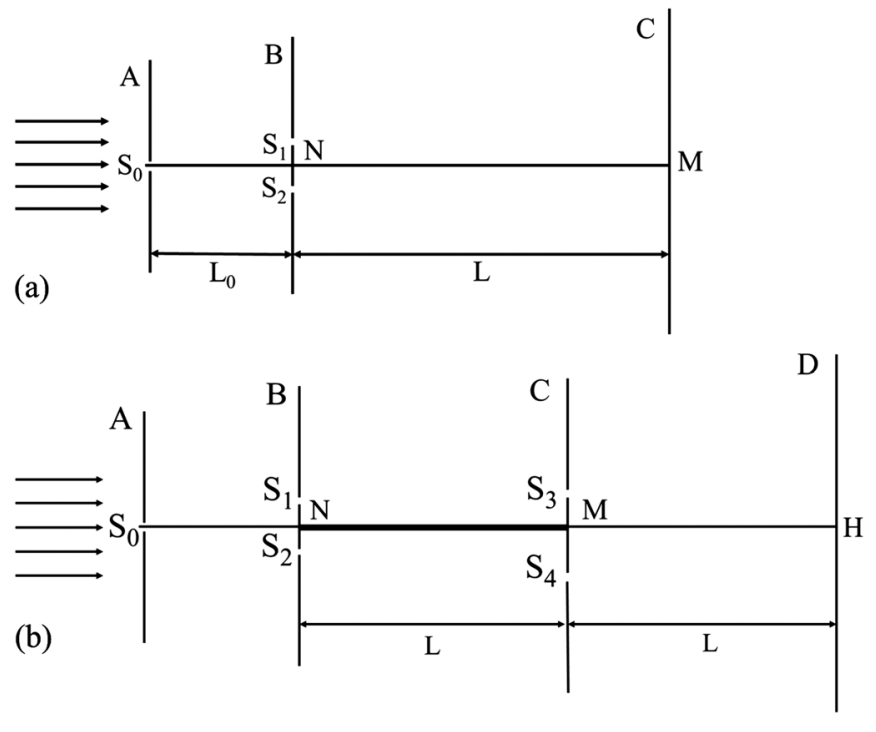

FIG. 6. (a) Q5 and Q6 problem setup. (b) Q7 and Q8 problem setup. In part(b), there is a barrier placed in between the first and second set of slits that blocks light from $S_{1}$ from entering $S_{4}$ and light from $S_{2}$ from entering $S_{3}$.

Question set 4 includes Q13-Q15 and focuses on standard Young's double slit questions (Fig. 2) with either the wavelength of the light source being changed (Q13 and Q14; typical contexts) or the medium being changed (Q15; atypical context). These questions were designed to test students' abilities to establish the connection through $\mathrm{CS} / \mathrm{CM} \rightarrow \mathrm{DW} \rightarrow \mathrm{PD} \rightarrow \mathrm{SW} \rightarrow \mathrm{IP}$.

Within set 2, students perform better on Q5 and Q6 than they do on Q7 and Q8, which is in line with the increase in difficulty of the concept application due to familiar and unfamiliar contexts (Table I). Out of the 41 students, 36 of them recognized that moving the screen would create a geometric path length difference and established the connection CP $\rightarrow$ PLD on Q5 and Q6. However, some of them then directly connected this to the interference pattern $(\mathrm{CP} \rightarrow \mathrm{PLD} \rightarrow \mathrm{IP})$ considering only the phase differences caused by the path difference after the double slits but not before. This led to 12 of the 36 students answering questions Q5 and Q6 incorrectly. Regarding Q7 and Q8, 23 of the 36 students applied the same approaches and about half (12 of the 23) answered these two questions correctly. Among those who did not answer correctly, 3 students believed that the only path difference necessary to be considered was between the second pair of slits and the screen, which is similar to the common incorrect reasoning observed in Q5 and Q6. Another 3 students separated the question in series and used the interference pattern created by the first set of slits at the position of the second set of slits as the coherent light sources for the second set. Two students ignored the second set of slits, treating these questions just as Q5 and Q6. The remaining students believe that the presence of the barrier blocks the 
light paths necessary to create an interference pattern and therefore surmised that no interference pattern would exist.

An additional question was posed to 9 interviewed students, removing the barrier from Q7 and Q8 such that there are two consecutive double slits. Three students believed that the final interference pattern would be the result of four light waves (from each of the four possible light pathways) interfering. Other students suggested that there would be no difference whether or not the barrier was there; or assumed that the first slit would not have an effect on the final pattern except by increasing the path lengths. One student correctly stated that the interference pattern at the second slits created by the first slits would determine the final pattern. The final pattern could be that of a single slit, double slit, or no pattern depending on if only one slit was at the location of a bright fringe, both slits, or no slits, respectively.

From the student data and interviews, it seems that students were able to establish the CP $\rightarrow$ PLD connection on typical questions but commonly were unable to connect further to the phase difference and superposition, instead skipping directly to the interference pattern. On atypical questions (Q7 and Q8), some students made partial logical analyses similar to how they approached the typical questions (Q5 and Q6) but were unable to fully grasp how concepts applied in novel situations. Much of this appears to stem from memorizing the standard double slit results without actual deep understanding of the central ideas. Attempts at answering atypical questions resulted in significant differences in both understanding of the central idea and applications of memorized materials. This suggests that both the conceptual understanding and the applications of certain memorized materials and problemsolving strategies are context dependent. Together, these are hallmarks of fragmented knowledge states.

Students' performances on question set 4 (Q13-15) are also similar. Overall, students did well on the typical questions (Q13, Q14) but scored poorly on the atypical question (Q15) as seen in Table I. Each problem focused on the same aspect of changing wavelength of light while differing how this change occurred. On the typical questions, 29 students were able to correctly establish the
$\mathrm{CS} \rightarrow \mathrm{DW}$ connection and apply relevant equations to determine the spacing between fringes.

For the atypical question (Q15), students used a wide variety of reasoning. Only five students applied the correct equation $\Delta y=\lambda L / n d$, while another 12 applied the same equation without $n$. Of the 12,7 then believed that $\Delta y$ did not depend on $n$ and therefore is unchanged, while the other 5 believed that because light travels slower in water that the wavelength shrinks as well, but they failed to advance further and apply this idea to solve the problem. The remaining 24 students simply guessed the answer and were not able to come up with any relevant reasoning or problem-solving strategies.

This set of questions again shows how typical and atypical contexts may influence student reasoning. The results suggest that many of the students are able to develop locally connected knowledge structures through memorization of equations and application to matched contexts. For these students, they were able to establish the CS $\rightarrow$ DW connection and use relevant equations to determine the spacing between fringes on familiar questions but failed to achieve a deeper understanding in order to establish $\mathrm{CM} \rightarrow \mathrm{DW}$ connection. Only a few students were able to achieve integrated deeper understanding and demonstrated the CS $\rightarrow$ $\mathrm{DW} \rightarrow \mathrm{PD} \rightarrow \mathrm{SW} \rightarrow \mathrm{IP}$ connection in problem solving.

\section{All atypical questions (question set 3)}

Question set 3 includes Q9-Q12, which are designed with all atypical contexts. The first two questions (Q9, Q10) alter the direction of the incident light so that they arrive at the slits with an angle and ask students to locate the zeroth bright fringe [Fig. 7(a)]. The remaining two questions (Q11, Q12) use a similar setup but ask students to find the phase difference at point $M$ on the screen [Fig. 7(b)]. These questions make emphasis on students' understanding of path difference and phase difference by introducing nonzero phase difference at the slits due to the angle of incoming light. The setups of all these questions are not usually taught, and therefore are considered atypical. This is evident from the low scores on these questions, similar to other atypical questions (Table I).

For Q9 and Q10, only 4 students correctly answered that the zeroth bright fringe was at $P$ and explained their

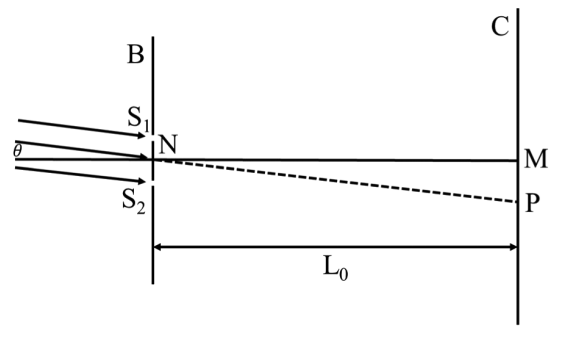

(a)

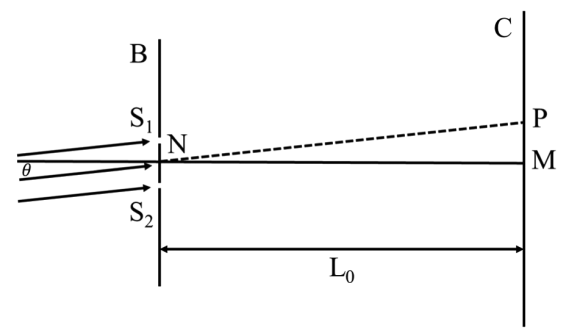

(b)

FIG. 7. (a) Q9 and Q11 problem setup. (b) Q10 and Q12 problem setup. 
reasoning that clearly showed the connection $\mathrm{CD} \rightarrow$ $\mathrm{IWPD} \rightarrow \mathrm{PD} \rightarrow \mathrm{SW} \rightarrow \mathrm{IP}$. Another 9 students picked the correct answer but used a variety of incorrect reasoning and believed there was no path difference created by the angle of the wave front at the slits. There were 12 students who ignored the angle of the incident light and believed that the zeroth bright fringe is located at $\mathrm{M}$, which is only true when the incident angle is zero (e.g., Q1). They did not think that the incident angle would create any additional path difference and only considered the path difference created in between the slits and the screen. The remaining students believed that the zeroth bright fringe would lie between point $M$ and point $P$ with a variety of reasoning, such as that the angle of the incident wave would create some initial phase difference but with uncertain amount and consequences.

The results of interviews on Q11 and Q12 are similar to that of Q9 and Q10. This time, a total of 8 students were able to answer the questions correctly with correct reasoning. 23 did not believe that the angle of the incident light would create a phase difference. The remaining 10 students recognized the initial difference but were unable to correctly reason with it further.

The results from Q9-Q12 indicate several levels of understandings among students. The top-level students were able to answer these questions correctly with correct reasoning. They demonstrated an integrated knowledge structure, with which they were able to apply the CD $\rightarrow$ IWPD $\rightarrow$ PD $\rightarrow$ SW $\rightarrow$ IP connection to solve questions with novel contexts. The intermediate level students were able to make some connections, specifically realizing that the incoming wave front would create a phase difference $(\mathrm{CD} \rightarrow$ IWPD); however, they were unable to apply this idea further to find the position of the zeroth fringe or final phase difference at a position on the screen. This reflects the lack of a connected understanding between an overall path difference and the total phase difference (PD), which is a core component of the central idea. Finally, the low-level students did not recognize that the angled wave front would create an initial phase difference but were split on how they approached finding the position of the zeroth fringe or final phase difference: the weakest students had no idea, while better students applied the same problem-solving method as in the basic case of Q1, indicating a memorized strategy.

\section{CONCLUSION}

Based on the qualitative and quantitative analysis of students' answers and reasoning on typical and atypical questions across the different content sets, three levels of student understanding can be identified. The best performing students appear to have developed a good understanding of the central idea and were able to apply it in reasoning to correctly answer most or all the typical and atypical questions. This level of students seems to have developed a well-integrated knowledge structure, with which they successfully recognized all aspects of the central idea (summarized in Fig. 1) and the possible connections to various contextual components. Their reasoning pathways used, as demonstrated through the interviews, could be applied across familiar and novel situations and were not context specific.

The medium performing students demonstrated effective reasoning in some familiar cases, but had significant difficulties applying the concept to novel situations. The difficulties of these students would manifest themselves differently for each student and context. However, a general theme emerged that the knowledge structures of the students were moderately fragmented with limited and isolated pathways connecting only some of the aspects of the central idea with familiar contexts. As a result, the conceptual understanding and problem-solving strategies of these students were highly context dependent with only established connections to typically learned contexts which were unable to transfer to any novel situations.

The final group were the worst performing students, who struggled on both typical and atypical questions. They may recognize some of the aspects of the concept and correctly answer questions based on memorized examples but were unable to establish a coherent reasoning which would demonstrate any form of deeper understanding.

These three categories of students, differentiated based on their performance on typical and atypical questions, can also be related to the "expert vs novice" model. The best performing students have demonstrated using the central ideas as the foundation to build connections among all the other physical elements to form a cohesive knowledge structure and are able to apply it consistently during problem solving, regardless of question contexts. This type of behavior is consistent with the expert behavior in the expert vs novice model. The medium performing students have shown that they made the appropriate connections when solving typical problems, while failing to do so when solving the atypical problems. This instability implies that they have some local connections within their knowledge structures but have not fully developed the connections leading through the central idea as the key pathways. This type of behavior is consistent with the transitional stage between expert and novice. The worst performing group merely relies on memorizing equations or examples that they have encountered previously, with most of the conceptual elements disconnected from one another. This is a demonstration of a significantly fragmented knowledge structure, with which the students may only recall memorized conceptual fragments and problem-solving strategies when familiar contexts are present. Therefore, when solving typical or atypical problems, these students usually make direct links from the given contexts and conditions to 
predict the outcome, without considering the central idea at all. This type of behavior is consistent with the novice behavior in the expert vs novice model.

Additionally, typical and atypical question designations are dependent upon instruction students receive and are not universal. It is expected, however, that with proper coding of typical and atypical questions, the groupings of novice, expert, and transitional students would exist in other education systems as well. Comparisons of different education settings and students' learning would be a valuable future step for research, in which the LIT can be a starting point of an assessment tool for research and teaching practices.

Furthermore, as is shown by this study, the learning behaviors of a significant fraction of Chinese students have also exhibited a lack a deep understanding and reliance on memorization for problem-solving strategies. These outcomes are similar to what has been found in U.S. students [7], despite the differences in the education settings between China and the U.S.A. Even though the Chinese physics curriculum put a strong emphasis on drilling in problem solving, most students are shown to still possess fragmented knowledge structures. This implies that experience in problem solving does not always transfer to develop a deep conceptual understanding. To remedy this issue so that students can obtain integrated knowledge structures, instructors can emphasize and clearly establish the central idea and develop connections through the central idea with the encountered conceptual components and contextual aspects. In practice, instructors can also demonstrate how to apply these connections in problem solving with wide ranging familiar and novel contexts.

Overall, teaching light interference could benefit from the additional focus paid to the conceptual framework. In theory, this would allow students to either form their initial knowledge structure around the central idea or connect preexisting pathways through the central idea to form an integrated knowledge structure. A similar study has recently been conducted demonstrating the benefits of this type of intervention while teaching force and motion concepts [33]. Further studies with different concepts as the focus would be required to determine the effectiveness of this intervention across broad contents.

\section{ACKNOWLEDGMENTS}

This work was supported by the NSF Grants No. DUE1044724, No. DUE-1431908, No. DRL-1417983, No. DUE-1712238, as well as the China Scholarship Council, Teacher Education Fund Project of Northeast Normal University (No. 131005020), and Teaching Reform Project in Higher Education of Jilin Province in P.R.C. Any opinions expressed in this work are those of the authors and do not necessarily represent those of the funding agencies.
[1] National Research Council, Research on Future Skill Demands: A Workshop Summary (National Academies Press, Washington, DC, 2008).

[2] National Research Council, Division of Behavioral and Social Sciences and Education, Board on Science Education, Board on Testing and Assessment, Committee on Defining Deeper Learning and 21st Century Skills, Education for Life and Work: Developing Transferable Knowledge and Skills in the 21st Century (National Academies Press, Washington, DC, 2012).

[3] D. Stamovlasis, T. Georgios, C. Kamilatos, D. Papaoikonomou, and E. Zarotiadou, Conceptual understanding versus algorithmic problem solving: Further evidence from a national chemistry examination, Chem. Educ. Res. Pract. 6, 104 (2005).

[4] M. H. Chiu, Algorithmic Problem Solving and Conceptual Understanding of Chemistry by Students at a Local High School in Taiwan, Proc. Natl. Sci. Counc. ROC(D) 11, 20 (2001).

[5] M. H. Chiu, C. Guo, and D. F. Treagust, Assessing students' conceptual understanding in science: An introduction about a national project in Taiwan, Int. J. Sci. Educ. 29, 379 (2007).
[6] E. Kim and S. J. Pak, Students do not overcome conceptual difficulties after solving 1000 traditional problems, Am. J. Phys. 70, 759 (2002).

[7] S. C. Nurrenbern and M. Pickering, Concept learning versus problem solving: Is there a difference?, J. Chem. Educ. 64, 508 (1987).

[8] B. S. Bloom, M. D. Engelhart, E. J. Furst, W. H. Hill, and D. R. Krathwohl, Taxonomy of Educational Objetives: The Classification of Educational Goals: Handbook I: Cognitive Domain (No. 373.19 C734t) (D. Mckay, New York, US, 1956).

[9] L. W. Anderson, D. R. Krathwohl, P. W. Airasian, K. A. Cruikshank, R. E. Mayer, P. R. Pintrich, J. Raths, and M. C. Wittrock, A Taxonomy for Learning, Teaching, and Assessing: A Revision of Bloom's Taxonomy of Educational Objectives, Complete Edition (Pearson, New York, 2000).

[10] M. Alonso, Problem solving vs. conceptual understanding, Am. J. Phys. 60, 777 (1992).

[11] A. B. Champagne, R. F. Gunstone, and L. E. Klopfer, A perspective on the differences between expert and novice performance in solving physics problems, Res. Sci. Educ. 12, 71 (1982). 
[12] M. T. Chi, P. J. Feltovich, and R. Glaser, Categorization and representation of physics problems by experts and novices, Cogn. Sci. 5, 121 (1981).

[13] P. T. Hardiman, R. Dufresne, and J. P. Mestre, The relation between problem categorization and problem solving among experts and novices, Memory Cognit. 17, 627 (1989).

[14] J. Larkin, J. McDermott, D. P. Simon, and H. A. Simon, Expert and novice performance in solving physics problems, Science 208, 1335 (1980).

[15] W. J. Gerace, R. J. Dufresne, W. J. Leonard, and J. P. Mestre, Problem Solving and Conceptual Understanding, in Proceedings of the 2001 Physics Education Research Conference, Rochester, NY, edited by K. Cummings, S. Franklin, and J. Marx (AIP, New York, 2001).

[16] B.-S. Eylon and F. Reif, Effects of knowledge organization on task performance, Cognit. Instr. 1, 5 (1984).

[17] A. H. Schoenfeld and D. J. Herrmann, Problem perception and knowledge structure in expert and novice mathematical problem solvers, J. Exper. Psychol. 8, 484 (1982).

[18] J. L. Snyder, An investigation of the knowledge structures of experts, intermediates and novices in physics, Int. J. Sci. Educ. 22, 979 (2000).

[19] H. S. Lee, O. L. Liu, and M. C. Linn, Validating measurement of knowledge integration in science using multiplechoice and explanation items, Appl. Meas. Educ. 24, 115 (2011).

[20] R. K. Sawyer, The Cambridge Handbook of the Learning Sciences (Cambridge University Press, Cambridge, England, 2005).

[21] J. Shen, O. L. Liu, and H. Y. Chang, Assessing students' deep conceptual understanding in physical sciences: an example on sinking and floating, Int. J. Sci. Math. Educ. 15, 57 (2017).

[22] H. Lee and O. L. Liu, Assessing learning progression of energy concepts across middle school grades: The knowledge integration perspective, Sci. Educ. 94, 665 (2010).

[23] M. Kubsch, J. Nordine, K. Neumann, D. Fortus, and J. Krajcik, Measuring Integrated Knowledge-A Network Analytical Approach, in Proceedings of the 13th International Conference of the Learning Sciences (ICLS) (London, United Kingdom, 2018).

[24] M. C. Linn, Internet Environments for Science Education (Routledge, London, 2013).

[25] N. B. Songer and M. C. Linn, How do students' views of science influence knowledge integration?, J. Res. Sci. Teach. 28, 761 (1991).

[26] J. Nordine, J. Krajcik, and D. Fortus, Transforming energy instruction in middle school to support integrated understanding and future learning, Sci. Educ. 95, 670 (2011).

[27] E. Alexopoulou and R. Driver, Small-group discussion in physics: Peer interaction modes in pairs and fours, J. Res. Sci. Teach. 33, 1099 (1996).

[28] C. H. Crouch and E. Mazur, Peer instruction: Ten years of experience and results, Am. J. Phys. 69, 970 (2001).

[29] C. Keller, N. Finkelstein, K. Perkins, S. Pollock, C. Turpen, and M. Dubson, Research-based practices for effective clicker use, AIP Conf. Proc. 951, 128 (2007).

[30] L. C. McDermott, Physics by Inquiry: An Introduction to Physics and the Physical Sciences (Wiley, New York, 1995), Vol. 1.
[31] K. J. Crippen and L. Archambault, Scaffolded inquiry-based instruction with technology: A signature pedagogy for STEM education, Computers in the Schools 29, 157 (2012).

[32] E. Gaigher, J. M. Rogan, and M. W. H. Braun, Exploring the development of conceptual understanding through structured problem-solving in Physics, Int. J. Sci. Educ. 29, 1089 (2007).

[33] Y. Nie, Y. Xiao, J. Fritchman, Q. Liu, J. Han, J. Xiong, and L. Bao, Teaching towards knowledge integration in learning force and motion, Int. J. Sci. Educ. (to be published).

[34] R. Duit and D. F. Treagust, Conceptual change: A powerful framework for improving science teaching and learning, Int. J. Sci. Educ. 25, 671 (2003).

[35] M.C. Linn, The knowledge integration perspective on learning and instruction, in The Cambridge Handbook of the Learning Sciences, edited by R. K. Sawyer (Cambridge University Press, Cambridge, England, 2005), pp. 243-264.

[36] L. Bao and E. F. Redish, Concentration analysis: A quantitative assessment of student states, Am. J. Phys. 69, S45 (2001).

[37] L. Bao and E. F. Redish, Model analysis: Assessing the dynamics of student learning, Phys. Rev. ST Phys. Educ. Res. 2, 010103 (2006).

[38] J. Minstrell, Facets of students' knowledge and relevant instruction, in Research in Physics Learning: Theoretical Issues and Empirical Studies, edited by R. Duit, F. Goldberg, and H. Niedderer (IPN, Kiel, 1992), pp. 110-128.

[39] B. S. Ambrose, P. S. Shaffer, R. N. Steinberg, and L. C. McDermott, An investigation of student understanding of single-slit diffraction and double-slit interference, Am. J. Phys. 67, 146 (1999).

[40] S. K. Şengören, How do Turkish high school graduates use the wave theory of light to explain optics phenomena?, Phys. Educ. 45, 253 (2010).

[41] S. K. Şengören, Turkish students' mental models of light to explain the single slit diffraction and double slit interference light: a cross-sectional study, J. Baltic Sci. Educ. 9, 61 (2010).

[42] V. Mešić, E. Hajder, K. Neumann, and N. Erceg, Comparing different approaches to visualizing light waves: An experimental study on teaching wave optics, Phys. Rev. Phys. Educ. Res. 12, 010135 (2016).

[43] B. S. Ambrose, P. R. L. Heron, S. Vokos, and L. C. McDermott, Student understanding of light as an electromagnetic wave: Relating formalism to physical phenomena, Am. J. Phys. 67, 891 (1999).

[44] A. Coetzee and S. N. Imenda, Alternative conceptions held by first year physics students at a south african university of technology concerning interference and diffraction of waves, Res. Higher Educ. J. 16, 1 (2012).

[45] L. Maurines, Geometrical reasoning in wave situations: The case of light diffraction and coherent illumination optical imaging, Int. J. Sci. Educ. 32, 1895 (2010).

[46] P. Colin and L. Viennot, Using two models in optics: Students' difficulties and suggestions for teaching, Am. J. Phys. 69, S36 (2001).

[47] M. Watts, Student conceptions of light: A case study, Phys. Educ. 20, 183 (1985).

[48] P. Hubber, Year 12 students' mental models of the nature of light, Res. Sci. Educ. 36, 419 (2006). 
[49] K. Wosilait, P. R. Heron, P. S. Shaffer, and L. C. McDermott, Addressing student difficulties in applying a wave model to the interference and diffraction of light, Am. J. Phys. 67, S5 (1999).

[50] K. Wosilait, Research as a guide for the development of tutorials to improve student understanding of geometrical and wave optics, Ph.D. thesis, University of Washington, 1996.

[51] V. Mešić, K. Neumann, I. Aviani, E. Hasović, W. J. Boone, N. Erceg, V. Grubelnik, A. Sušac, D. S. Glamočić, M. Karuza, A. Vidak, A. Alihodžić, and R. Repnik, Measuring students' conceptual understanding of wave optics: A Rasch modeling approach, Phys. Rev. Phys. Educ. Res. 15, 010115 (2019).
[52] R. D. Sokoloff, Active Learning in Optics and Photonics Training Manual, Paris: UNESCO, 2006.

[53] G. A. Morris, L. Branum-Martin, N. Harshman, S. D. Baker, E. Mazur, S. Dutta, T. Mzoughi, and V. McCauley, Testing the test: Item response curves and test quality, Am. J. Phys. 74, 449 (2006).

[54] See Supplemental Material at http://link.aps.org/ supplemental/10.1103/PhysRevPhysEducRes.15.020134 for the LIT items and the related psychometric analysis.

[55] Y. Xiao, J. Han, K. Koenig, J. Xiong, and L. Bao, Multilevel Rasch modeling of two-tier multiple choice test: A case study using Lawson's classroom test of scientific reasoning, Phys. Rev. Phys. Educ. Res. 14, 020104 (2018). 